\title{
The value of clinics for the relief of chronic pain
}

\author{
Mark Swerdlow North West Regional Pain Relief Centre, Hope Hospital, Manchester
}

This paper is another of the papers presented at a conference on Pain organised under the auspices of the London Medical Group. Mark Swerdlow deals with the work of pain relief clinics and explores their value. He gives the background as to what these clinics are, describes who is treated, how they are staffed and finally offers his opinion as to their effectiveness.

The phrase the 'value of clinics for the relief of chronic pain' might be taken as a reference to cost effectiveness. Leaving aside the relief of human suffering I believe it would be possible to show that pain relief clinics are good value for money, but I am going to take the word 'value' to refer simply to clinical effectiveness. This in fact should be rather easier to prove than its cost-effectiveness because for one thing many of the patients are old (retired) and many are housewives. They have had all the conventional treatments and seen all appropriate specialists and despite this (sometimes perhaps because of this) they have intractable pain. If a proportion of these patients leave the clinic pain-free - or with a level of pain with which they can cope - I think it reasonable to conclude that pain relief clinics have a value.

\section{What is a pain relief clinic?}

It is a curious fact that although descriptions of severe pain can be found in writings of 2000 and more years ago, medical and surgical text books of the 18th and even 19th Centuries have very little to say about the relief of pain. The first clinic ever opened solely for the treatment of patients with chronic pain was a small nerve blocking clinic opened in 1942 in New York. A recent census, showed there are now at least 325 major Pain Centres and these are situated as follows (Table I):

The pain relief clinic has in fact four different functions:

I) Diagnostic - although a diagnosis has usually been made before the patient is referred, some of the most difficult cases present difficult diagnostic problems.
2) Therapeutic
3) Teaching
4) Research

I will talk about these latter two presently.
Table I Census of pain relief centres

\begin{tabular}{lc}
\hline Country & No. of pain relief centres \\
\hline America & 225 \\
Japan & 20 \\
Great Britain & 15 \\
Canada & 15 \\
Italy & 9 \\
Germany & 6 \\
Australia & 5 \\
Others & 30 \\
\hline Total & 325 \\
\hline
\end{tabular}

\section{Whom and what do we treat?}

Most clinics see patients referred by both GPs and by hospital specialists. The ideal treatment of pain is of course to remove the cause and the thing which $\vec{\theta}$ pain clinic patients have in common is that the cause po of their pain is undiscovered or untreatable conditions such as cancer, post-herpetic neuralgia amputation stump pain, trigeminal neuralgia, causalgia and lumbo-sciatic syndrome.

Curiously enough some of the best results are obtained in patients with cancer pain - partly because the patient has a limited length of life left and therefore relief has to last for only a limited time and partly because the cause is more 'mechanical' than in many pains of benign origin.

I have told you that many of the patients who arrive at the clinic have a feeling they have been 'discarded' that 'nothing more can be done'. But 3 'Learn to live with it' is easier for the doctor than

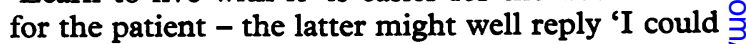
live with it easily if it were your pain, Doctor'. A common advantage of treatment at the clinic is improvement in function. As the years go by in $\frac{D}{0}$ unrelieved patients the pain can result in disuse atrophy in a hand, arm or leg. When the pain is $\mathcal{N}$ reduced or relieved it is sometimes possible to $N$ produce considerable improvement in movement. N Some of the cases which have given me most ${ }_{\sigma}^{\omega}$ pleasure have been not only because we have relieved pain but that we have also given the patient ${ }^{\circ}$ a useful limb.

\section{Who staffs a pain relief centre?}

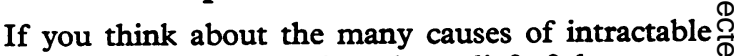
pain it must be quite clear that relief of these sorts $\stackrel{\mathbb{Q}}{\circ}$ 
of conditions will call on the expertise of a number of different kinds of specialist - for example cancer pain may need a radio-therapist or an oncologist, some central pains call for neurosurgery, backache involves an orthopaedic surgeon and the demoralisation which often accompanies chronic pain can be helped by a psychiatrist, while some peripheral and not-so-peripheral neuralgias can be relieved by the anaesthetist with neurolysis applied either by injections, radiofrequency current or the cryoprobe. The modern concept of a pain relief clinic or centre is therefore a multi-disciplinary one. In addition to the various specialists, the nursing staff, the physiotherapy department and the medical social workers all have an important part to play in the clinic's activities.

The internal arrangement varies from clinic to clinic, and to some extent depends on the nature of the work and the types of specialities. In some centres the specialists jointly consult on many of the patients; in others patients are referred from specialist to specialist as necessary and 'case conferences' are held only on difficult cases which baffle the individual specialists. With all patients after a certain length of time if no progress is being made another colleague is consulted or a case conference is arranged. The important thing is that the patient arrives at the most effective form of treatment and in the least possible number of 'moves'.

It is important that the patient's family doctor is kept fully in the picture.

\section{Effectiveness}

Why should the pain clinic be more successful than the hitherto conventional methods of relieving pain ?

r) Because the pain centre provides all currently available methods of treating chronic pain, both invasive and non-invasive, physical, pharmacological and psychological;
2) Because the personnel devote a greater or lesser portion of their time to dealing with care of intract- $\propto$ able pain; they are conversant with what the other members of the team can do and with current advances in the field and not least, they have ? empathy for the patient;

3) As with all specialisation, by concentrating the treatment of certain conditions in a specialist centre the members get far more experience than the individual clinicians from whom the cases are referred. This concentration of cases of course $O$ facilitates the organisation of research into improved in methods of treatment; it also makes the centre an $\overrightarrow{0}$ ideal place to teach students, both undergraduate and post-graduate, about the problems in intractable $\bar{\omega}$ pain and the principles and methods of treatment.

It is important that one member of the team remains responsible for each patient and that the patient knows who his doctor is.

Some would argue that pain relief clinics gather $\vec{v}$ hysterics, scroungers and malingerers - I should 의 have thought that having a multidisciplinary clinic would be the best way of avoiding this.

There is also an argument that patients with conditions such as chronic osteroathritis and $\stackrel{\mathbb{D}}{3}$ spondylosis should suffer - suffering ennobles treating pain makes people soft. I don't believe treatment for bona fide pain makes people soft $\sigma \varphi$ especially when the treatment hurts! But in practie if the clinic does not treat them someone will and perhaps not as expertly.

We do not relieve all the patients by any means. Pain is a crutch on which to lean; it is a cry for help and the cry needs interpreting - sometimes the $\stackrel{\mathbb{Q}}{\mathbb{2}}$ need is not for medicine, sometimes the cause is $\overrightarrow{\vec{A}}$

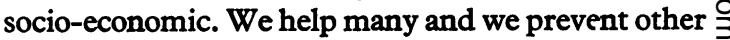
from succumbing to escalating drugs or neurosurgical operations - and there is every hope that we will do better still. I think therefore that pain relieving clinics are of value. 